\title{
Pobreza, vulnerabilidad y calidad de vida en América Latina. Retos para la bioética
}

Francisco Javier León Correa ${ }^{1}$

Resumen: Trabajo de revisión bibliográfica sobre los retos para la bioética en Latinoamérica. Se exponen primero algunos datos éticamente relevantes sobre la pobreza y la dependencia en Latinoamérica. Después se analiza la noción de pobreza y vulnerabilidad desde la filosofía y la respuesta ética que exige de nosotros, para examinar finalmente las repercusiones de los datos previos y de la reflexión filosófica sobre pobreza, vulnerabilidad y calidad de vida en la bioética latinoamericana especialmente.

Palabras clave: pobreza, vulnerabilidad, calidad de vida, bioética

Poverty, vulnerability and quality of life in Latin America. Challenges for bioethics

\begin{abstract}
This article is a bibliographical review about challenges for bioethics in Latin America. First, some ethically relevant data about poverty and dependence in Latin America are presented. Afterwards, poverty and vulnerability notions are analyzed from a philosophical perspective and ethical response required, ending by examining the consequences of previous data and philosophical reflection about poverty, vulnerability and quality of life, especially in Latin American bioethics.
\end{abstract}

Key words: poverty, vulnerability, quality of life, bioethics

\section{Pobreza, vulnerabilidade e qualidade de vida na América Latina. Desafíos para a bioética}

Resumo: Trabalho de revisão bibliográfica sobre os desafíos para a bioética na América Latina. Primeiro são expostos alguns dados eticamente relevantes sobre a pobreza e a dependência na América Latina. Depois se analisa a noção de pobreza e vulnerabilidade a partir da filosofia e da resposta ética que é exigida de nós para examinar finalmente as repercussóes dos dados prévios e da reflexão filosófica sobre pobreza, vulnerabilidade e qualidade de vida na bioética latino-americana especialmente.

Palavras-chave: pobreza, vulnerabilidade, qualidade de vida, bioética

\footnotetext{
${ }^{1}$ Doctor en Filosofía, Magíster en Bioética. Profesor Adjunto, Centro de Bioética, Facultad de Medicina, Pontificia Universidad Católica de Chile, Chile

Correspondencia: fleon@uc.cl
} 


\section{Introducción}

El artículo $8^{\circ}$ de la Declaración Universal sobre Bioética y Derechos Humanos de la UNESCO, aprobada en 2005, establece que "al aplicar y fomentar el conocimiento científico, la práctica médica y las tecnologías conexas, se debería tener en cuenta la vulnerabilidad humana. Los individuos y grupos especialmente vulnerables deberían ser protegidos y se debería respetar la integridad personal de dichos individuos"(1). Sin entrar en el término utilizado en potencial, "debería", que más bien debiera ser imperativo - "se debe" o, mejor, "debemos" (2)—, es claro que la protección de los individuos y grupos más vulnerables es una de las misiones principales de la bioética en su reflexión sobre los valores en la atención y en los sistemas de salud.

La vulnerabilidad es la situación de dependencia en que pueden estar personas o grupos sociales, que no permite o pone en riesgo la autodeterminación y la libre elección en sus ideales de vida y en su desarrollo. Podemos hablar de varios niveles de vulnerabilidad. En primer lugar, la que tenemos todos por el hecho de ser humanos: nuestra libertad no es absoluta, estamos condicionados - a veces coaccionados- de modo interno o externo, y en concreto todos enfermamos y finalmente morimos.

Un segundo nivel, que es el que importa en este trabajo, consiste en no tener cubiertas las necesidades básicas, por la pobreza o la falta de salud, que son términos casi sinónimos en muchas situaciones concretas de Latinoamérica. No nos referimos aquí a las situaciones de extrema pobreza y de extrema gravedad por una enfermedad, pues en estos casos, más que de personas vulnerables hablamos de personas vulneradas en su condición humana $y$, por lo tanto, con una calidad de vida muy por debajo de lo deseable. Ante esto, el deber ético es procurar cuanto antes devolver a esas personas a situaciones de dignidad, en el caso de la pobreza, o acompañar y procurar la mejor "calidad de vida" posible, aunque lo que de verdad procuramos es la "mejor calidad de muerte", con cuidados paliativos, porque ya están fuera del alcance terapéutico.
Nos referimos a las situaciones de pobreza no extrema y de falta de salud, por enfermedades crónicas o con posibilidades de curación, que afectan en mayor o menor medida la calidad de vida de las personas y que suponen, también en mayor o menor medida, una situación de vulnerabilidad, de posibilidad bastante próxima de dependencia de otros y de no autodeterminación. En este sentido sí que podemos hablar de situaciones, personas o grupos sociales especialmente vulnerables, a los que se debe procurar una mejora en su "calidad de vida", promoviendo que dispongan de una mejor salud y de más medios económicos, culturales y educativos.

Desde los pobres y enfermos, receptores de la ayuda de los demás, podemos hablar de "derechos de bienestar" y, sobre todo, del deber de promover su autonomía. Desde los agentes que pueden ayudar, los principios éticos fundamentales son los de justicia y solidaridad.

"Posiblemente la mayor tragedia biológica que sufre la especie humana es que su población crece con individuos vulnerados en su vitalidad, no ya sólo existencialmente vulnerables"(3), en un mundo cada vez más cargado de riesgos y sobre todo de miedo, como señala Bauman(4), consecuencias más directas de la vulnerabilidad.

Veremos primero algunos datos acerca de la pobreza y la dependencia en Latinoamérica que nos parecen éticamente relevantes, antes de entrar en el análisis bioético. Después, analizaremos la noción de pobreza y de vulnerabilidad desde la filosofía, y la respuesta ética que exige de nosotros, para exponer finalmente las repercusiones, en la bioética latinoamericana especialmente, de estos datos previos y de esta reflexión filosófica sobre pobreza, vulnerabilidad y calidad de vida.

Tabla 1: Pobreza en América Latina

\begin{tabular}{|l|l|l|l|l|l|l|l|l|l|}
\hline & 2000 & 2001 & 2002 & 2003 & 2004 & 2005 & 2006 & 2007 & 2008 \\
\hline $\begin{array}{l}\text { Extrema- } \\
\text { pobreza }\end{array}$ & 18,1 & 18,5 & 19,4 & 10,1 & 16,9 & 15,4 & 13,3 & 12,6 & 12,9 \\
\hline Pobreza & 42,5 & 43,2 & 44,0 & 44,2 & 42,2 & 39,8 & 36,5 & 34,1 & 33,0 \\
\hline
\end{tabular}


Desigualdad entre los que tienen más y los que tienen menos

Hemos mostrado los datos de pobreza en América Latina de la Comisión Económica para América Latina y El Caribe (CEPAL), recogidos entre 1989 y 2004(5), para ver la relación del ingreso medio per cápita entre el quintil 1 y el quintil 5, que tiene en cuenta los hogares del conjunto de cada país ordenados según su ingreso per cápita. El quintil 5 representa el 20\% de los hogares de más altos ingresos, en tanto que el quintil 1 representa el 20\% de los hogares de menores ingresos.

La media de la región entre la primera medición de cada país y la última muestra que la desigualdad sigue creciendo: 21,0 y 21,9 . Aunque son menores las cifras totales de extrema pobreza y de pobreza, la separación entre lo que tienen más y los que tienen menos ha aumentado. En la última medición están claramente por debajo de la media, con mayor igualdad relativa, Uruguay $(10,2)$, México, Perú, Argentina, Ecuador y Costa Rica. Y están muy por encima de la media Bolivia $(44,2)$, Brasil $(31,8)$ y Colombia $(29,6)$.

\section{Discapacidad}

En América Latina viven aproximadamente 85 millones de personas con discapacidades, de las cuales sólo $2 \%$ encuentran respuestas a sus necesidades, según datos del Programa Regional de Rehabilitación de la Organización Panamericana de la Salud (OPS). Los objetivos de las políticas de la OPS en este campo son los siguientes(6):

- Garantizar la protección social en salud para todos los ciudadanos.

- Contribuir e eliminar las desigualdades en el acceso.

- Garantizar servicios de calidad.

- Proporcionar oportunidades para recibir atención integral a los grupos sociales excluidos.

- Eliminar la capacidad de pago como factor restrictivo.

Las causas principales de la discapacidad en América Latina son las sanitarias: además de las comunes con los países desarrollados (defectos congénitos, enfermedades crónicas, tumores malignos, accidentes de tránsito, entre otras), están las enfermedades infecciosas y parasitarias, deficiencias nutricionales y problemas en el embarazo y el parto. También tienen importancia las ambientales: la contaminación ambiental y su repercusión en la salud, la falta de prevención de la violencia y de los accidentes laborales y de tránsito, y los conflictos armados en algunos países, que causan traumas psicológicos y emocionales. Podemos concluir que son áreas problemáticas la educación, el empleo, la accesibilidad y movilidad en áreas urbanas, el poco desarrollo e idoneidad de la asistencia médica para la discapacidad y la falta de información.

\section{Trastornos mentales en América Latina}

Es preciso conocer también la prevalencia de los trastornos mentales y la brecha de tratamiento, dada por la diferencia entre las tasas de prevalencia verdadera y las de personas efectivamente tratadas, que en algunos casos es grande, pese a la existencia de tratamientos eficaces(7). Los resultados de una amplia investigación basada en estudios comunitarios publicados en toda América Latina y el Caribe es la siguiente(8): las psicosis no afectivas (entre ellas la esquizofrenia) tuvieron una prevalencia media estimada durante el año precedente de 1,0\%, la depresión mayor de 4,9\% y el abuso o dependencia del alcohol de 5,7\%. Más de la tercera parte de las personas afectadas por psicosis no afectivas, más de la mitad de las afectadas por trastornos de ansiedad y cerca de tres cuartas partes de las que abusaban o dependían del alcohol no habían recibido tratamiento psiquiátrico alguno, ya en un servicio especializado o en uno de tipo general.

\section{Los determinantes sociales de la salud}

Es patente que las desigualdades en salud crean en ocasiones desigualdades sociales, pero también su contrario se ha puesto de manifiesto: cada vez está más fundado que las desigualdades sociales crean desigualdades en salud(9), por lo que es necesario tenerlas muy presentes a la hora de establecer políticas de atención y de buscar propuestas y mejoramientos(10). 
"De la merma de años de vida ajustados por discapacidad, el $80 \%$ es sufrida por las naciones más pobres. Hay múltiples datos epidemiológicos que confirman la profunda brecha de salud y expectativas de vida que se da entre pudientes y desposeídos"(3:47). Los espectaculares logros alcanzados por la salud de los individuos, con la mejoría mayoritaria de las expectativas de vida, por ejemplo, no han sido equiparados por una mejoría equivalente de la salud de las poblaciones en su conjunto, por lo que debemos llamar la atención "hacia los valores dominantes que han moldeado nuestro mundo (...) y hacia una ética de la salud pública que considere tanto los derechos como las necesidades humanas" (11:195), dentro de "los compromisos necesarios de una medicina sostenible"(12).

No podemos enfocar el análisis bioético sólo en los derechos de los pacientes desde el ámbito clínico, debemos profundizar en los aspectos éticos de la salud pública y, más aún, en los determinantes sociales de las deficientes situaciones de salud.

\section{Calidad de vida}

La pobreza y la discapacidad se relacionan —en ocasiones muy directamente- con la enfermedad, y las tres con la "calidad de vida". Este término empieza a utilizarse en la década de los 60 y como categoría específica de las ciencias sociales en los 70. Se ha convertido en un elemento esencial de la atención en salud: "los sistemas de salud en general, y cada situación clínica en particular, procuran aumentar la calidad de vida de las personas enfermas, que han de asumir determinadas limitaciones para poder prolongar su vida. $Y$ en aquellos casos en que el pronóstico apunta hacia una muerte más o menos inminente, se trata de optimizar la calidad del tiempo que reste por vivir"(13).

Se han desarrollado diferentes técnicas de evaluación de la calidad de vida con instrumentos más o menos precisos, como los ańos de vida ajustados por calidad (QALYs) o por discapacidad (DALYs), que han sido incorporados al análisis bioético(14), y la calidad de la asistencia en salud también se intenta medir con criterios concretos evaluables (15).

El concepto de calidad de vida ha sido un elemento de estudio relevante en los problemas de bioética al final de la vida, en la distribución justa de los recursos en salud y a la hora de tomar decisiones en la mayoría de los problemas de salud pública, de manera que elevar la calidad de vida de los pacientes comprende un amplio abanico de propósitos y puede estar presente como el fin último del sistema de salud de un país y como el objetivo de cada tratamiento a un paciente en particular. Quizás no sea el fin último, pero sin duda es uno de los principales fines de la medicina actual y de las políticas públicas de salud.

\section{Pobreza desde la filosofía política}

Desde una concepción ética de la pobreza, ser pobre significa no contar con determinados recursos económicos, pero también, y sobre todo, carecer de lo que se ha denominado la autoestima o el respeto propio, carencia que va en contra de los mínimos "derechos de bienestar" destinados a proporcionar ayuda para salir de ella. "Como señala A. Sen, las poblaciones pobres son desempoderadas, carecen de la capacidad para hacer uso de la libertad, con el fin de incorporarse a la sociedad y participar en su desarrollo. Es imposible abordar temas de ética que conciernen a la humanidad entera sin registrar la ingente realidad de la pobreza que golpea notoria y visiblemente a casi la mitad de la población mundial" (3:74-75).

Según Rawls, la pobreza afecta a los valores morales primarios: "Las bases sociales del respeto de sí mismo son aquellos aspectos de las instituciones básicas que normalmente son esenciales para que los ciudadanos tengan un sentido vivo de su propio valor como personas morales y sean capaces de realizar sus intereses de orden supremo y promover sus fines con confianza en sí mismos" (16).

Existen muy diversas posiciones acerca de cómo afrontar la pobreza y sobre los criterios de distribución de la riqueza que afectan a las posibles políticas públicas. Así, el criterio de distribución según los bienes primarios de $\operatorname{Rawls(17),~o~la~}$ 
conocida sentencia de la doctrina marxista: "De cada cual según su capacidad, a cada cual según sus necesidades" (18), centrados ambos criterios en el principio de justicia. El problema es cómo atender con medios escasos necesidades crecientes y evaluar de modo objetivo estas necesidades. Se proponen entonces listas de necesidades básicas(19) o atender mejor a las necesidades subjetivas según las preferencias de los afectados, como propone Dworkin(20). Con los criterios de preferencias, el centro comienza a variar desde la justicia y los derechos objetivos a la autonomía personal. Marta Nussbaum habla de "actualizar las potencialidades" de los seres humanos(21). Onora O'Neill ha insistido en que la pobreza sitúa a las personas en una situación de vulnerabilidad que les impide rechazar lo que les ofrecen aquellos que detentan el poder(22). Amartya Sen subraya que cualquier teoría sobre la justicia debe tener como fin el desarrollo de "las capacidades y los funcionamientos" que permiten a las personas participar en la vida social: la pobreza es, sobre todo, privación de las capacidades y de la libertad de agencia para el bienestar(23).

Desde otras posiciones, más ligadas al principio ético de beneficencia que al de estricta justicia, se insiste en las obligaciones de asistir: desde el consecuencialismo utilitarista, Singer comenta que "si podemos evitar algo malo, sin sacrificar algo que tenga un valor moral comparable, debemos hacerlo"(24), y está claro que la pobreza es lo peor en un nivel social.

Otros insisten en que más allá de los derechos y las obligaciones están las virtudes (sobre todo la justicia entendida como virtud), que llevan a los individuos a organizar la solidaridad y emprender acciones de lucha contra la pobreza, y que "las instituciones encargadas de satisfacer las necesidades básicas tengan una actitud activa y responsable y no se conformen con sentir, pasivamente, que cumplieron con la demanda de un derecho" (25).

\section{Vulnerabilidad desde la filosofía}

La vulnerabilidad, en la visión filosófica, y especialmente en la personalista, es una de las justificaciones del hecho mismo de la moralidad. Entre las características constitutivas del ser humano - junto a la insuficiencia del instinto, la racionalidad, la autonomía, la responsabilidad y la índole comunitaria - está precisamente la vulnerabilidad: "nuestra vulnerabilidad no queda plenamente amparada por las solas prescripciones negativas de la moralidad. Ésas marcan los mínimos. Necesitamos también de las contribuciones positivas de otras personas: intimidad, cariño, protección y cuidado. La satisfacción de estas necesidades solamente es posible en una comunidad de seres morales, en la que se reconocen y amparan las vulnerabilidades y los intereses de las personas"(26).

Los seres humanos somos "animales racionales y dependientes". Como expone MacIntyre: "Cuando se habla en los libros de filosofía moral de los enfermos o de quienes padecen alguna lesión o sufren alguna discapacidad, se les trata casi exclusivamente como individuos que pueden ser objeto de benevolencia por parte de los agentes morales, quienes aparecen en cambio como sujetos continua y constantemente racionales, con buena salud y que no padecen alteración alguna" (27). Así los "discapacitados" son "ellos", diferentes de nosotros, personas en cuya situación nos hemos visto alguna vez o nos vemos ahora, o probablemente nos veremos en el futuro.

La vulnerabilidad y la dependencia son universales y nos llevan a una necesaria cooperación social fundada en las virtudes personales. Las virtudes que el ser humano necesita para desarrollarse y llegar a ser un agente racional e independiente son las mismas que las que requiere para hacer frente a la vulnerabilidad y la discapacidad, tanto de uno mismo como de los demás. Son "las virtudes propias de los animales racionales y dependientes" (28), por lo que la razón misma nos invita a una filosofía humana del cuidado de la persona vulnerable(29).

Pero estas virtudes no pertenecen solamente al ámbito privado; deben hacerse presentes también en los ámbitos social y político, como "compromiso ciudadano que lleve a una articulación política de la solidaridad"(30) y a una discriminación jurídica positiva de los más dependientes y vulnerables(31). 


\section{Elaboración de una ética del cuidado y la soli- daridad}

"La vulnerabilidad tiene el poder de sacar lo mejor de nosotros mismos, activar los valores más sólidos, ayudarnos a construir redes basadas en la relación y no en la independencia, basadas en el interés por los demás y no sólo por uno mismo. En el modo como nos acercamos a la vulnerabilidad propia y ajena revelamos nuestro grado de humanidad"(32).

Es necesario por ello elaborar una ética del cuidado que, más allá de los debates actuales sobre los límites de la justicia, se acerque a la dimensión personal de la experiencia de la discapacidad y la vulnerabilidad, de forma que lleve a una mayor acción dentro de la justicia social completada con la solidaridad(33). Este tema ha cobrado mayor actualidad a la vista de las recientes pandemias(34) y de las consecuencias de las catástrofes naturales.

"Es preciso reconocer que la ética del cuidado pone de relieve elementos importantísimos para la vida moral. El cuidado se refiere, en definitiva, a la sensibilidad ante las necesidades y vulnerabilidades del otro concreto, en su singularidad, con quien estamos en relación"(23). La ética filosófica debe descender al trato que se debe proporcionar a quienes se hallan en situación de dependencia, desde las éticas clásicas a las éticas de la responsabilidad y, finalmente, a las del cuidado, claves para analizar "las repercusiones que comporta para la ética médica la extensión social de personas en situación de especial vulnerabilidad(35). Y, desde la ética, debemos aplicar esta perspectiva en la bioética y la biojurídica(36), de modo que no se centren exclusivamente en las relaciones de derechos y deberes, sino también en construir relaciones éticas que fortalezcan en lo posible la vida frágil de las personas(37).

Más allá de las alternativas entre deontologismo y teleologismo, principialismo o consecuencialismo, es necesario promover una praxis ética que reivindique el derecho y deber de todo ser humano a una vida digna, a partir de los cauces epistemológicos que identifican bioética y desarrollo solidario(38).

\section{Desde Latinoamérica: propuesta desde la éti- ca del discurso sobre la pobreza y el desarrollo económico y social}

Dorando J. Michelini ha elaborado una detallada propuesta desde la ética discursiva: "Las problemáticas de la pobreza y la exclusión, que se han agravado en América Latina en las últimas décadas, presentan también serios desafíos para la realización del bien común"(39). Por eso, en los últimos ańos, la cuestión del desarrollo y de una ética del desarrollo se vuelven a plantear con fuerza dentro de las éticas prácticas $(40,41)$ y de la ética de la empresa y la economía(42).

Pero ya no se plantea este desarrollo con una concepción economicista y meramente técnica. "En la actualidad, al hablar de desarrollo, es necesario tener en cuenta la integralidad de un proceso que incluye lo económico, lo ecológico y lo cultural, como así también la dimensión ético-política. No hay desarrollo sin eficiencia económica", pero ésta debe estar "ético-políticamente orientada al bien común, y articulada con la identidad cultural y el cuidado del medio ambiente, sobre la base de una corresponsabilidad e interacción solidarias, tal como lo exigen los lineamientos fundamentales de una nueva ética cívica" (43).

\section{Los pobres en la sociedad del conocimiento}

Gilberto Cely llama a la reflexión sobre la gran responsabilidad que incumbe a la ciencia y la tecnología - y al científico en su trabajo cotidiano individual - en la configuración de la nueva sociedad, la sociedad del conocimiento, en la que los pobres son precisamente los marginados culturalmente(44). La nueva sociedad del conocimiento tendría que caracterizarse por la solidaridad entre quienes la conforman, que ayude a superar los fraccionamientos tradicionales.

Precisamente por esto, varios de los encuentros académicos sobre bioética han tenido el objetivo de profundizar en la vulnerabilidad que supone la falta de educación y de conocimientos, como una "problemática que consideramos de apremiante interés para los países de la región (...) (y) como aporte a la discusión y a la construcción de una 
verdadera 'ciudadanía social', con una visión integradora de los distintos ámbitos del saber que confluyen en el ámbito de la bioética” (45).

\section{Efectiva aplicación de los derechos humanos}

Con fundamentos en esta ética dialógica, pero más allá de esa reducción de las asimetrías de los interlocutores, parte de la ética y de la bioética latinoamericana se ha centrado en dos temas: cómo conseguir una efectiva aplicación de los derechos humanos, especialmente el derecho a la vida y el derecho a la asistencia justa en salud, y cómo conseguirlo en una región donde son muy numerosas las personas vulnerables y las que ya han sido vulneradas en algunos de sus derechos más fundamentales, tanto por la pobreza y falta de desarrollo social y económico, como por la marginación y exclusión étnica o social.

La preocupación por los derechos humanos está en el inicio de las primeras publicaciones de temas de bioética en México, por ejemplo, con las publicaciones del Instituto de Investigaciones Jurídicas de la UNAM en los años ochenta, que han continuado hasta la actualidad(46). También son recientes las visiones globales de la bioética como una ética de los derechos humanos, sobre todo como una profundización del principio de justicia del principalismo, con la equidad como fundamental por encima de la eficiencia del sistema de salud, por ejemplo(47), o en abierta crítica a este sistema de principios(48).

Para ello, la erradicación de la pobreza y el desarrollo económico y social son el primer paso imprescindible. Algunas propuestas van en la línea de apoyar la equidad en las reformas de los sistemas de salud, con parámetros bien delimitados, como la de Norman Daniels(49,50). Otras van directamente a la fundamentación: la justicia se entiende como solidaridad y directamente en la lucha contra la exclusión derivada del neoliberalismo, como comenta Pérez de Nucci: "La justicia es el nombre que la ética biomédica propone como nueva filosofía de la salud para la consideración y tratamiento del problema de los pobres"(51).

\section{Una bioética de liberación e intervención}

Es bien conocida la fundamentación de Dussell de una ética de la liberación, que parte de la crítica a la ética dialógica, por considerar que el diálogo racional se efectúa con gran disparidad en Latinoamérica: no es un diálogo entre iguales(52) y las "formas de vida intersubjetivamente compartidas" parten de injusticias radicales, por lo que la ética debe ser primero una ética de la liberación de los oprimidos(53). Si para Levinas la ética era la filosofía primera, para Dussel esa filosofía primera es la política, una ética-política de liberación que evite el silenciamiento de los oprimidos(54), colmo de la "asimetría antidialógica".

En bioética, esta corriente se ha desarrollado sobre todo en Brasil $(55,56)$ y en algunos casos ha dado lugar a la elaboración de propuestas de una bioética de intervención(57), directamente en el campo político, y de reforma de los sistemas de salud. Sin embargo, marcando las distancias entre bioética y política como campos complementarios, otras posiciones se esfuerzan en distinguirlas: "Si la política se adscribe a la deliberación en forma de una democracia ética dispuesta a legitimar su proceder, estará en un terreno común y fructífero con la bioética. Es menester que la política reconozca sus raíces éticas y la proveniencia moral de su legitimidad, más que pedirle a la bioética que intente desarrollar un discurso político"(58).

\section{Bioética de protección de los vulnerables}

También desde Brasil y Chile ha surgido la propuesta de una bioética de protección de los vulnerados y empoderamiento de los vulnerables(59): "La bioética de protección, entendida como un nuevo abordaje que debe ser aplicado en los países en desarrollo, quiere, sobre todo, normativizar las prácticas humanas, anticipando e incentivando los efectos positivos y con previsión de los eventuales efectos dañinos para los individuos y los pueblos, de modo que se prescriban los actos considerados correctos y se proscriban los incorrectos, dentro de la realidad latinoamericana (...), con niveles de desigualdad vergonzosos y con dolencias propias del subdesarrollo aliadas a patologías de países desarrollados"(60). 
La propuesta más detallada de una ética de la protección es, hasta ahora, la de $\operatorname{Kottow}(61)$ : "Reconociendo las inequidades existentes, la ética de protección se propone paliarlas mediante el desarrollo de actitudes personales de amparo y programas sociales de resguardo (...) este texto desarrolla la estructura de un ética de protección —individual y colectiva-, a fin de ponerla a disposición de una protección bioética, es decir, una bioética que entienda su cometido como una abogacía por los más débiles y una herramienta conceptual que brega por el uso de instituciones sociales que fomenten el empoderamiento de los postergados".

\section{Conclusión}

Una visión bioética de la calidad de vida en Latinoamérica no puede ser sólo un modo de determinar la proporcionalidad o no de un tratamiento médico, o de delimitar desde la economía los grados de pobreza o riqueza de algunos grupos sociales distribuidos en quintiles. Lo que nos importa es la situación de vida que deben tener los seres humanos, lo cual se nos presenta como una exigencia moral a cada uno, pues los otros y su vida nos interpelan —en el sentido de Ricoeur y Levinas-y nos plantean exigencias desde la ética personal y, sobre todo, desde la ética social.

La calidad de vida es parte de la dignidad de la persona y, por ello, es una finalidad — quizás la primera- de la medicina y de las políticas públicas de salud. En ella se fundamenta un deber ético de ayudar a los demás para que dejen de estar vulnerados en sus derechos y necesidades básicas, para que sean menos vulnerables y para que dis- fruten de la mejor situación posible de libertad y de vida autónoma plena.

Más allá de una bioética centrada en asegurar la no maleficencia y el respeto a los derechos de justicia por parte de todos, absolutamente imprescindible en nuestros países, debemos avanzar en el respeto de la autonomía de las personas, profesionales de la salud y pacientes. Y parte de este respeto es el deber ético de promover su competencia como agentes morales, mediante una solidaridad personal, institucional y social que lleve a la inserción social de los más vulnerables y a conseguir su efectivo empoderamiento para llevar adelante sus ideales de vida buena, con una solidaridad y una beneficencia no paternalistas.

La bioética como interdisciplina puede ayudar a enfrentar los temas más apremiantes en nuestros países, que hacen referencia a "la equidad en el acceso a bienes y servicios, la necesidad de armonizar tradiciones diversas en la superior síntesis del panamericanismo, la constante necesidad de reformar y transformar instituciones (...) temas convertidos en procesos sociales, ideas fuerza y esfuerzo legislativo"(62).

Esto supone políticas sociales que concreten la solidaridad — que ésta no se ejerza sólo particularmente- y la organización de unos sistemas de salud con atención de calidad, más allá de las atenciones básicas, que supere las desigualdades en el acceso y en las prestaciones de salud. Papel de la bioética es impulsar estos valores en la reflexión de la deontología profesional, de la ética institucional en salud y de la ética pública en las políticas y sistemas de salud. 


\section{Referencias}

1. UNESCO. Declaración Universal de Bioética y Derechos Humanos; 2005.

2. Cárdenas R. La DUBDH de la UNESCO: Consideraciones en torno a sus alcances y naturaleza jurídica. En: Abellán J, et al. Temas de Bioética y Derecho. Lima: Cátedra UNESCO de Bioética y Biojurídica, Facultad de Derecho UNIFE; 2008: 71-95.

3. Kottow M. Bioética ecológica. Bogotá: Universidad El Bosque; 2009: 71.

4. Bauman Z. Miedo líquido. Barcelona: Paidós; 2007.

5. CEPAL. Proyecto: "Seguimiento del componente de pobreza del primer objetivo de desarrollo del Milenio (ODM)". Base de datos Pobreza en América Latina de la Comisión Económica para América Latina y el Caribe, División de Desarrollo Social. Disponible en: http://websie.eclac.cl/sisgen/ConsultaIntegrada.asp?idAplicacion=14)

6. Amate AE, Vásquez AJ, (eds.) Discapacidad: lo que todos debemos saber. Washington: OPS/OMS; 2006: 6.

7. Rodríguez JJ, Khon R, Aguilar-Gaxiola S. Epidemiología de los trastornos mentales en América Latina y el Caribe. Washington: OPS; 2009.

8. Kohn R, Levav I, Caldas de Almeida JM, et al. Los trastornos mentales en América Latina y el Caribe: asunto prioritario para la salud pública. Revista Panamericana de Salud Pública 2005; 18(4/5): 229-240.

9. Emanuel E. Inequidades, bioética y sistemas de salud. En: Lolas F, (ed.) Bioética y cuidado de la salud. Equidad, calidad, derechos. Santiago de Chile: Programa Regional de Bioética, OPS/OMS; 2000: 101-106.

10. Construyendo la nueva agenda social desde la mirada de los determinantes sociales de la salud. Documento síntesis del Ciclo de Diálogos Democráticos. Santiago de Chile: OMS/OPS, Ministerio de Salud Chile, FLACSO Sede Chile; 2005: 96-105.

11. Benatar SR. Public health and public health ethics. Acta Bioethica 2003; 9(2): 195-207.

12. Callaham D. La inevitable tensión entre la igualdad, la calidad y los derechos de los pacientes. En: Lolas F, (ed.) Bioética y cuidado de la salud. Equidad, calidad, derechos. Santiago de Chile: Programa Regional de Bioética, OPS/OMS; 2000: 91-100.

13. García Viniegras CV. Calidad de vida. Aspectos teóricos y metodológicos. Buenos Aires: Paidós; 2008: 37.

14. Gracia D. Ética de la calidad de vida. En: Gracia D. Fundamentación y enseñanza de la bioética. Bogotá: El Búho; 1998: 155-173.

15. Simón P, (ed.) Ética de las organizaciones sanitarias. Nuevos modelos de calidad. Madrid: Triacastela; 2005.

16. Rawls J. Unidad social y bienes primarios. En: Justicia como equidad. Materiales para una teoría de la justicia. Madrid: Tecnos; 1986: 193.

17. Rawls J. Teoría de la Justicia. México: Fondo de Cultura Económica; 1995.

18. Heller A. Theory of Needs in Marx. London: Allison and Bubsy; 1974.

19. Nussbaum N, Sen A, (comps.) La calidad de vida. México: Fondo de Cultura Económica; 1996.

20. Dworkin R. Do Liberty and Equality Conflict? En: Barker P, (comp.) Livings as Equals. Oxford: Oxford University Press; 1996: 23-57.

21. Nussbaum M. Capacidades humanas y justicia social. Una defensa del esencialismo aristotélico. En: Riechman J, (coord.) Necesitar, desear, vivir. Sobre necesidades, desarrollo humano, crecimiento económico y sustentabilidad. Madrid: Los Libros de la Catarata; 1998: 43-104.

22. O’Neill O. Toward Justice and Virtue. Cambridge: Cambridge University Press; 1998.

23. Sen A. Bienestar, justicia y mercado. Barcelona: Paidós; 1997.

24. Singer P. Practical Ethics. 2a ed. Cambridge: Cambridge University Press; 1994: 229.

25. Dieterlen P. La pobreza: un estudio filosófico. México: UNAM y Fondo de Cultura Económica; 2006: 111. 
26. Ferrer JJ, Álvarez JC. Para fundamentar la bioética. Teorías y paradigmas teóricos en la bioética contemporánea. 2a ed. Bilbao: Universidad Pontificia de Comillas y Desclée de Brouwer; 2005: 44-45.

27. MacIntyre A. Animales racionales y dependientes. Por qué los seres humanos necesitamos las virtudes. Barcelona: Paidós Básica; 2001: 10-11.

28. Sánchez Escorial L, Verona Humet P. Identidad, justicia y vulnerabilidad. Madrid: Fundación Mapfre Medicina, 2005: 19.

29. Masiá Clavel J. El animal vulnerable: invitación a la filosofía de lo humano. Madrid: Universidad Pontificia de Comillas; 1997.

30. Pérez Tapias JA. Del bienestar a la justicia. Aportaciones para una ciudadanía intercultural. Madrid: Trotta; 2007: 118-127.

31. Alarcos FJ. ¿Vivir dependiendo? Ética, derecho y construcción biográfica en la dependencia. Granada: Comares; 2007.

32. Bermejo JC, (ed.) Cuidar a las personas mayores dependientes. Santander: Sal Terrae; 2002.

33. Ralston DCh, Ho J, (eds.) Philosophical reflections on Disability. Netherlands: Springer Verlag; 2010.

34. Brody H, Avery E. Medicine’s Duty to Treta Pandemic Illness: Solidarity and Vulnerability. Hasting Center Report 2009; 39(1): 40-48.

35. Bonete E. Etica de la dependencia. Madrid: Editorial Tecnos; 2009.

36. Cayuela A, (coord.) Ética, bioética y desarrollo: el hombre como ser dependiente. Granada: Comares; 2004.

37. Del Barco JL. La vida frágil. Buenos Aires: EDUCA, Instituto de Bioética; 2000.

38. De Velasco JM. La bioética y el principio de solidaridad. Una perspectiva desde la ética teológica. Bilbao: Universidad de Deusto; 2003.

39. Michelini DJ. Bien común y ética civica. Una propuesta desde la ética del discurso. Buenos Aires: Bonum; 2008: 71.

40. Martínez Navarro E. Ética para el desarrollo de los pueblos. Madrid: Trotta; 2000.

41. Goulet D. Ética del desarrollo. Guía teórica y práctica. Madrid: IEPALA; 1999.

42. Cortina A, Pereira G, (eds.) Pobreza y libertad. Erradicar la pobreza desde el enfoque de Amartya Sen. Madrid: Tecnos; 2009.

43. Conill J. Horizontes de economía ética. Aristóteles, Adam Smith, Amartya Sen. Madrid: Tecnos; 2006: 72 .

44. Cely Galindo G. La bioética en la sociedad del conocimiento: los pobres del Tercer Milenio son los que no acceden al conocimiento. Bogotá: 3R Editores; 1999.

45. Hooft PF, Chaparro E, Salvador H, (comps). Bioética, vulnerabilidad y educación. VIII Jornadas Argentinas y Latinoamericanas de Bioética. Mar del Plata: Ediciones Suárez; 2003.

46. Tealdi JC. Bioética de los derechos humanos. Investigaciones biomédicas y dignidad humana. México: Instituto de Investigaciones Jurídicas, UNAM; 2008.

47. Martínez Oliva L. Justicia y medicina pública. Del principio ético a los desafíos en su aplicación. Santiago de Chile: LOM Ediciones; 1999.

48. Estévez E, García Banderas A. Bioética de intervención. Los derechos humanos y la dignidad humana. Quito: Terán; 2009.

49. Daniels N, et al. Benchmarks of fairness for health care reform: a policy tool for developing countries. Bull World Health Organ 2000; 78(6): 740-750.

50. Daniels N. Benchmarks of Fairness for Health Sector Reform in Developing Countries: Overview and Latin American Applications. Disponible en http://www.paho.org/English/BIO/Daniels.ppt

51. Pérez de Nucci A. Antropoética del acto médico. Tucumán: Facultad de Filosofía y Letras, Universidad Nacional; 2008: 115.

52. Dussel E. Ética de la liberación en la edad de la globalización y de la exclusión. Madrid: Trotta; 2008.

53. Dussel E. Ética del discurso y ética de la liberación. Madrid: Trotta; 2005. 
54. Romero Cuevas JM. Humanismo, historia y crítica en Ignacio Ellacuría. En: Rüsen J, Kpzlarek O, (coords.) Humanismo en la era de la globalización. Desafíos y perspectivas. Buenos Aires: Editorial Biblos; 2009: 129-144.

55. Moser A, Soares AM. Bioética. Do consenso ao bom senso. Petrópolis, Brasil: Editora Vozes; 2006.

56. Fabri dos Anjos M, De Siqueira JE, (orgs.) Bioética no Brasil. Tendencias e perspectivas. São Paulo: Sociedade Brasileira de Bioética, Ideias Letras; 2007.

57. Garrafa V, Porto D. Bioética de intervención. En: Tealdi JC, (director). Diccionario Latinoamericano de Bioética. Universidad Nacional de Colombia (Sede Bogotá), UNESCO Red Latinoamericana y del Caribe de Bioética; 2008: 177-180.

58. Kottow M. Bioética y biopolítica. Bioética, Sociedade Brasileira de Bioética 2005; 2: 110-121: 121.

59. Schramm FR, Kottow M. Principios bioéticos en salud pública: limitaciones y propuestas. Cadernos de Saude Pública 2001; 17.

60. Schramm FR, Rego S, Braz M, Palacios M, (orgs.) Bioética, riscos e proteçâo. Río de Janeiro: Editora Fiocruz; 2005: 17.

61. Kottow M. Ética de protección. Una propuesta de protección bioética. Bogotá: Universidad Nacional de Colombia y Escuela Salud Pública de la Universidad de Chile; 2007.

62. Lolas F. Hacia una Bioética para América Latina y el Caribe. En: Lolas F, (ed.) Bioética y cuidado de la salud. Equidad, calidad, derechos. Santiago de Chile: Programa Regional de Bioética, OPS/ OMS; 2000: 13-18.

Recibido: 15 de junio de 2010

Aceptado: 13 de septiembre de 2010 